ISAHP 2001, Berne, Switzerland, August 2-4, 2001

\title{
VOICE ROUTING AND DELAY PROBLEM IN DATA NETWORKS
}

\author{
Mehmet Kara ${ }^{1}$ \\ Computer Engineering \\ Kocaeli University \\ Vinsan Kocaeli-Turkey \\ mehmet29@hotmail.com \\ mkara@uekae.tubitak.gov.tr \\ Huseyin Karabelli ${ }^{2}$ \\ Computer Engineering \\ Kocaeli University \\ Vinsan Kocaeli-Turkey \\ karabelli@kou.edu.tr \\ Engin Demiray $^{3}$ \\ Computer Engineering \\ Kocaeli University \\ Vinsan Kocaeli-Turkey \\ hedemiray@kou.edu.tr
}

Keywords: QoS, Voice, delay, segmentasyon, Prioritisation, smoothing delay

Summary:Today Routing techniques are required to guarantee the various quality of service (QoS) characteristic requested by packed switched Networks. However it is known that such a routing algorithms, with two or more add itive or multicaptive QoS matrics in any possible combinations is NPcomplete. In this paper we propose delay problem and its effect on voice transmission. We exemined with motorola vanguard router QoS metrics. These metrics are link speed, data packet length, packed priority, variable delay and smooting delay. We choose link speed and packed length then we measured the packed delay. For each link we determined the what shoulde be packed length.

\section{Introduction}

Today PSTN networks uses TDM which reserve dedicated timeslots/Bandwidth to transport voice packets. When voice connection set up between two node voice, noise and silence is transmitted in this channel. For data traffic, both data and flags are transmitted. Thus available bandwidth is used inefficiently. On the other hand when we use STDM sending voice or data information We don't send flag, noise and silence. When we sent voice STDM offers some challenge for continuous bite rate low delay traffic. Some of voice packets can not be reach destination node on time because of the data transmission. This delay effect voice quality.

In traditional data networks, routing protocols usually characterize the network with a single metric such as hop-count or delay. Shortest-path algorithms are then used in these networks for path computation. The subjectivity of Quality of Service (QoS) requirements of the diverse traffic class in multimedia networks and complex trade-offs among them make it difficult to define an appropriate unique routing metric. It is known that such a routing problem involving two or more additive or multiplicative QoS parameters in any possible combination is NP-complete (Jaffe, 1984; Wang, Crowcroft, 1996). Hence number of heuristic algorithms have been recently proposed to solve this problem. In heuristic approaches 
the complexity of the QoS routing algorithms is reduced by choosing a subset of QoS parameters- there are no dynamic routing methods that use a complete set of QoS parameters to determine a route for multimedia data flows (Vogel, Herrtwivh,1996). One possible approach is to define a function and generate a single metric for multiple parameters. For example (Wang, Croft,1996) proposed a composition rule by choosing a bottleneck bandwith and propagation delay as a routing metric. Iwata (Iwata, Izmailov, Lee, Sengupta, Ramamurthy, Suziki,1996) used a criterion which optimize the network resource utilization of path satisfying multiple QoS requirements of users, while limiting both call blocking probability and the connection setup delay to a sufficiently low value.

\section{The Delay Problem and Its Main Cause}

By way of example let us assume that the voice DSP in the diagram generates 50 voice packets per second and that each packet must be sent to the Frame relay port for transmission as soon as it is generated. The voice packet will be encapsulated in Frame relay or TCP/IP and sent over the WAN to its destination. The destination DSP will receive the packet and must then convert it back into analogue speech. At 50pps it is evident that each voice packet will generate $20 \mathrm{msecs}$ of speech. When the $20 \mathrm{msecs}$ of speech has been sent, the receiving DSP will expect the next voice packet to be available for processing - but what if it has not yet arrived? In this case the DSP can not send any speech to its attached telephone until the next voice packet arrives. This leads to short 'gaps' in the received analogue signal and gives an effect referred to as 'choppy speech'. The diagram shows a situation in which the transmission of one or more speech packets is certain to be delayed. Suppose, for example, that a voice packet has been transmitted and the node starts to transmit a long data packet (e.g. a 1500byte IP datagram). At $64 \mathrm{Kbps}$ it will take around $188 \mathrm{msecs}$ to transmit 1500 bytes, so that if a voice packet is waiting it will have to wait for anything up to $188 \mathrm{msecs}$ before it can be sent. Clearly some method is required to ensure that voice packets are transmitted as quickly as possible, which will include some method of controlling, or minimizing, the time a voice packet must wait in a Frame Relay port or TCP/IP transmission queue. The following notes will explain how this is achieved in the

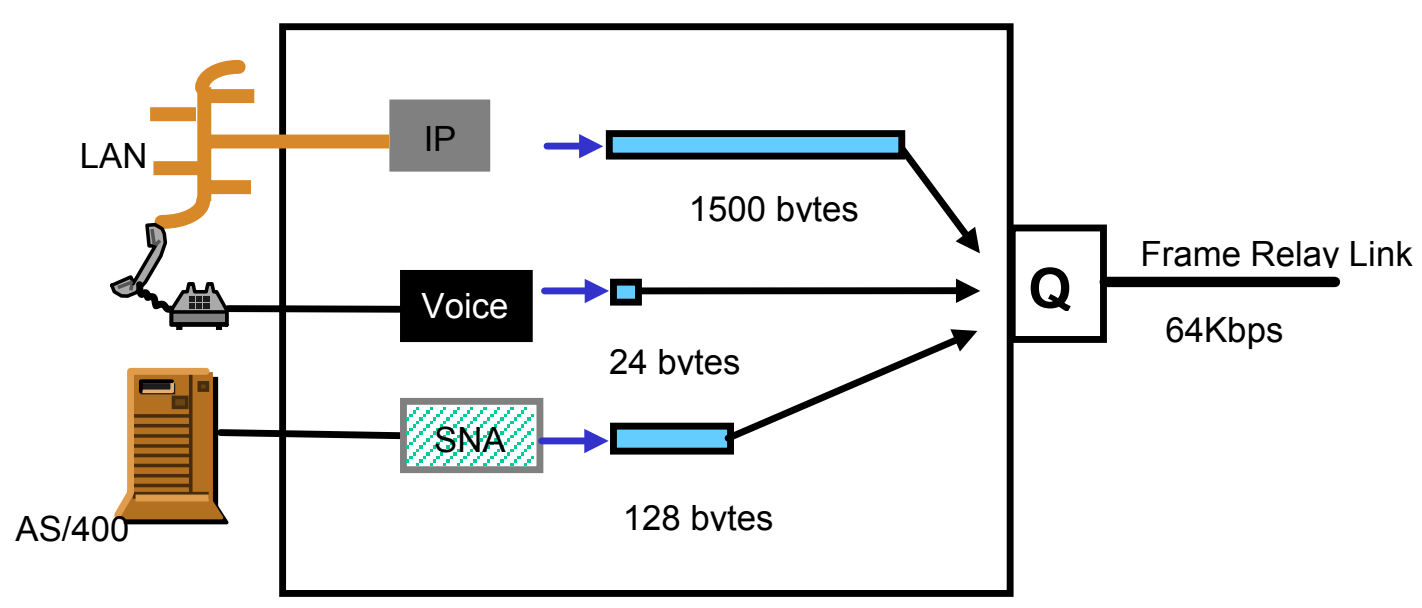

Figure-1. Packet Transmission

\subsection{Traffic Prioritization}

The first step in dealing with the voice packet transmission delay problem is to implement some form of traffic prioritisation scheme. Following example explain this cause, let us assume that we have voice, IP data, and SNA data all 'contending' for transmission out of the same Frame Relay WAN port. Clearly they must first form an orderly queue for transmission. 
- Rule 1: Voice traffic is always assigned highest transmission priority. This is very logical because voice packets are always short. At $64 \mathrm{Kbps}$, for example, it takes only 3 to 4 milliseconds to transmit a complete voice packet - it is not holding other traffic up for very long.

- Rule 2: Other traffic (e.g. IP and SNA) can be assigned transmission priority during router configuration. This allows users to ensure that their time-critical, interactive applications will achieve good response times. The configurable priority systems featured in the Vanguard

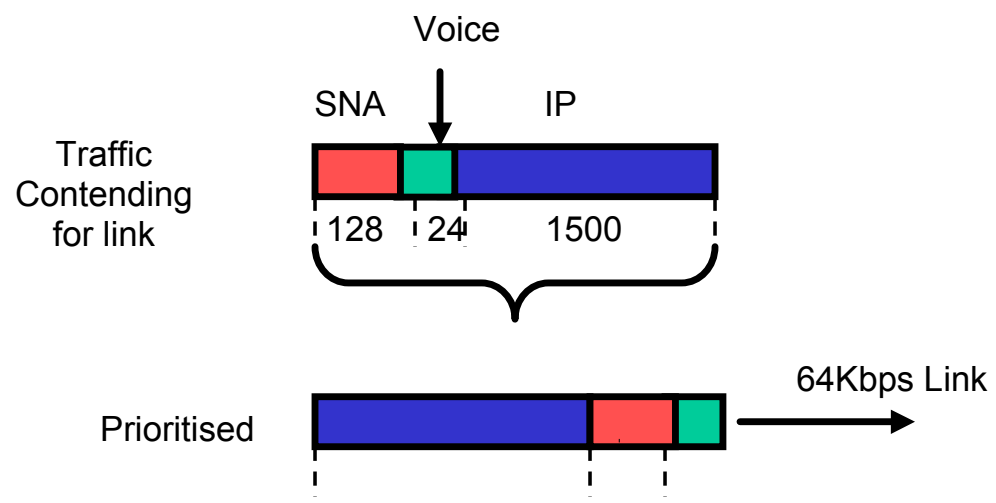

Figure-2. Packet Prioritization

Prioritization alone is not enough because the voice packet is sent first and takes approximately 3 msecs to transmit. The 128byte SNA packet is sent second and takes approximately $16 \mathrm{msecs}$ to transmit. Now we transmit the IP packet, and it will take around $188 \mathrm{msecs}$ to complete send. BUT, if the voice DSP is generating 50 voice packets per second (I.e. one packet every $20 \mathrm{msecs}$ ) a new voice packet will appear just after we have started to send the IP packet. This means that the new voice packet will be badly delayed - and we can guarantee that the receiving voice will contain gaps of silence (choppy voice).

\subsection{Packet Segmentation}

Segmentation is a process of splitting long data packets into a number of smaller 'fragments' or 'segments' - as shown in the figure 3 . The whole purpose of segmentation is to ensure that Voice packets will always be transmitted within a known period of time. To explain this the router operation will be described:

- Segmentation can be enabled or disabled for each Frame Port in the router configuration. Only those FR ports which carry voice packets should have fragmentation enabled.

- The segment size is also configurable. For example, that it is set to 64 bytes. This means that a 1500 byte IP packet will be segmented into 24 segments, and a 128 byte SNA packet will become 2 segments. Voice packets will never be segmented because they are already shorter than 64 bytes.

-But now there will be a new routing rule:

- A Voice packet will never be queued behind more than two data segments

- The queuing (transmission) delay problem is now solved. If a voice packet can never be queued behind more than two data segments then, in this example, a voice packet might be queued while 128 bytes ( 2 segments) are being transmitted. But it only takes around $16 \mathrm{msecs}$ to send 128 bytes at $64 \mathrm{Kbps}$, so every voice packet will be transmitted as soon as it is generated.

- Dynamic Segmentation:

- Segmentation is done dynamically. Segmentation will operate on a FR port only if voice packets are being transmitted. When there are no voice packets, segmentation is automatically switched off. This is increase efficiency of connection. 


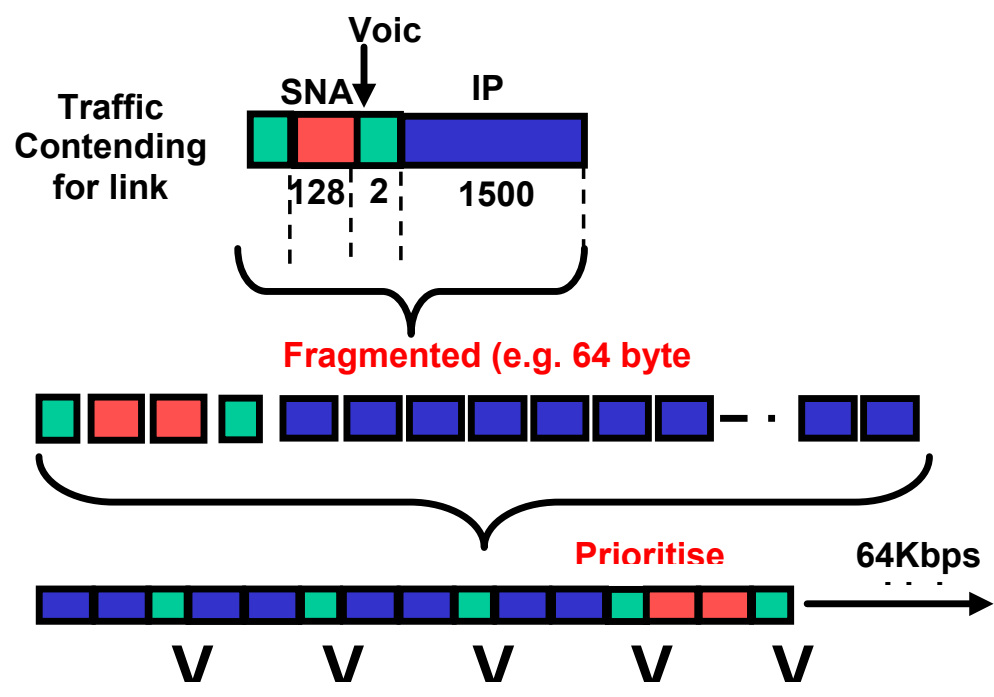

Figure-3. Packet Segmentation

\subsection{Variable Delay}

Even with segmentation and traffic prioritisation it can not always be guaranteed that voice packets will arrive at the destination DSP within the required time period. Both voice and data are multiplexed over the FR network. At the source, voice packets are continuously generated while a person is talking. On the network link, voice packets are multiplexed with other forms of traffic. This multiplexing causes gaps between successive voice packets. At the remote end, the individual traffic streams are de-multiplexed. When the voice is played out, there are gaps between successive samples which results in "choppy voice". Consider a DSP which generates $50 \mathrm{pps}$ of voice when it is transmitting. Voice packets are therefore transmitted at strict $20 \mathrm{msec}$ intervals. In theory, then, if a voice packet arrives at the destination and is processed the NEXT voice packet should arrive $20 \mathrm{msecs}$ later if we are to avoid the 'choppy' speech problem. Unfortunately this can not be guaranteed, so we need a solution. Variable delay is directed related to the size and number of intervening packets which are multiplexed between succeeding voice packets

\subsection{Smoothing Delay}

The solution is to provide the router Voice card with a configurable Smoothing Buffer. When the first voice packet arrives it is not processed immediately but is held in the Smoothing Buffer for a configured period of time, called the smoothing delay time. If, for example, the smoothing delay time is set to 40 msec then the first voice packet will not be processed for $40 \mathrm{msec}$. After $40 \mathrm{msec}$ has elapsed the DSP will process the first voice packet and generate $20 \mathrm{msec}$ of speech. The second packet "should" already be available so it will be processed next to provide another $20 \mathrm{msecs}$ of speech. The end-result is this if the Smoothing Delay is set to $40 \mathrm{msec}$ then all will work well provided that each voice packet arrives within $40 \mathrm{msec}$ of the previous voice packet.

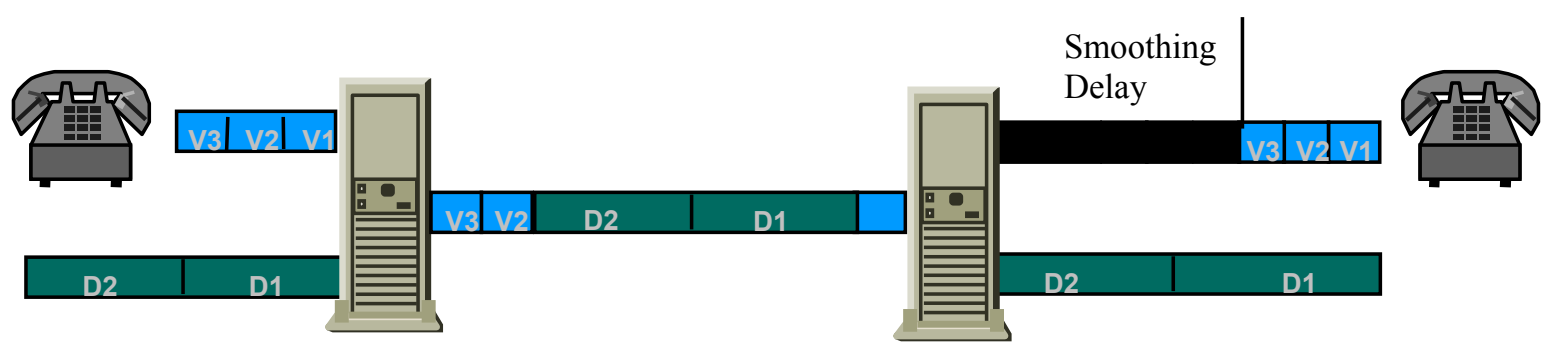

Figure-4. Smoothing Delay 


\section{Selecting the Segment Size}

The Table will help you to select the optimum segment size for a given Frame relay link speed. The table shows the time it takes to transmit different segment sizes at different link speeds.

To select the optimum segment size:

- Run down the link speed column until you come to the speed of the FR link

- Run across the columns until you find the shaded items

- Note that the segment size can be different if the network is a basic point-to-point setup, or a multi-hop setup.

-Maximum Queuing Delay (MQD) Algorithm:

- $\mathrm{MQD}=\{(2 \mathrm{x}$ delay of largest data packet $)+($ delay of the number of voice channels- 1$)\}$

\begin{tabular}{|l|l|l|l|l|l|l|l|}
\hline $\begin{array}{l}\text { Link } \\
\text { speed } \\
\text { kbps })\end{array}$ & $\begin{array}{l}\text { 32 Byte } \\
\text { Data } \\
\text { Packets }\end{array}$ & $\begin{array}{l}\text { 64 Byte } \\
\text { Data } \\
\text { Packets }\end{array}$ & $\begin{array}{l}\text { 128 Byte } \\
\text { Data } \\
\text { Packets }\end{array}$ & $\begin{array}{l}\text { 256 Byte } \\
\text { Data } \\
\text { Packets }\end{array}$ & $\begin{array}{l}\text { 512 Byte } \\
\text { Data } \\
\text { Packets }\end{array}$ & $\begin{array}{l}\text { 1024 Byte } \\
\text { Data } \\
\text { Packets }\end{array}$ & $\begin{array}{l}\text { 1500 Byte } \\
\text { Data } \\
\text { Packets }\end{array}$ \\
\hline 19.2 & 18,3 & 31.7 & 58.3 & 111.7 & 218.3 & 431.7 & 630 \\
\hline 28.8 & 12,2 & 21.1 & 38.9 & 74.4 & 145.6 & 287.8 & 420 \\
\hline 56 & 6,3 & 10.9 & 20 & 38.3 & 74.7 & 148 & 216 \\
\hline 64 & 5,5 & 9.5 & 17.5 & 33.5 & 38.3 & 129.5 & 189 \\
\hline 128 & 2,8 & 4.8 & 8.8 & 16.8 & 65.5 & 64.8 & 94.5 \\
\hline 256 & 1,4 & 2.4 & 4.4 & 8.4 & 32.8 & 32.4 & 47.3 \\
\hline 384 & 1 & 1.6 & 2.9 & 5.6 & 10.9 & 21.6 & 31.5 \\
\hline 512 & 0,7 & 1.2 & 2.2 & 4.2 & 8.2 & 16.2 & 23.6 \\
\hline 1024 & 0,2 & 0.4 & 0.7 & 1.4 & 2.7 & 5.4 & 7.8 \\
\hline 2048 & 0,2 & 0.3 & 0.5 & 1.0 & 2.0 & 4.1 & 5.0 \\
\hline
\end{tabular}

\section{Conclusion}

In this study we proposed voice and data packed delays on the packed switching networks. We want to transport our voice information on the data networks. But the main problem guarantee QoS. In this study we determined the main problems of voice transmitting and QoS requirements. We determined packed delay according to given link speed, packed length. Choosing proper parameters we obtained good improvements in voice quality.

\section{References}

Iwata, R. Izmailov, D. Lee, B. Sengupta, G. Ramamurthy, and H. Suziki. (1996) “ATM Routing Algorithms with multiple QoS Requrements for Multimedia Internetworking” IEICE Transections on Communications E79-B

W.C. Lee, M.G. Hluchyi, and P.A. Humble.(1995) "Routing Subject to Quality of Service Constraint in Integrated Communication Networks" IEEE Network

T. McMullen, (1999), “Vanguard 6425 Router Manual”, Massachusetts, US

M. Schwartz, (1987) Telecommunication Networks:Protocols, Modeling and Analysis, Addison Wesly, Reading

E. Aboelela, C. Douligeris (1998), "Fuzzy Inference System For QoS Routing in B-ISDN," IEEE Canadian Conference on Electrical and Computer Engineering (CCECE 98), Waterloo, Canada pp.141144 
I.Chlamtac, A. Farago, H. Zhang (1998), "A Deterministic Approch to the End to End Analysis of Packet Flows in Connection Oriented Networks," Transactions on Networking, Agust

Z. Wang, J.Crowcroft (1996), "Quality of Service Routing for supporting multimedia Applications," IEEE Jurnal on Selected Area in Communications Vol 14, pp.1228-1234

R.L. Cruz, “A Calculs for Network Delay Part I network Elements in Isolation,” IEEE Trans. Inform. Teory, Vol.37, pp.114-131, Jan 1991 